\section{Proposta de modelo hierarquizado aplicado à investigação de fatores de risco de óbito infantil neonatal}

\author{
Proposal for a hierarchical framework applied \\ to investigation of risk factors for \\ neonatal mortality
}

\footnotetext{
${ }_{1}$ Escola Nacional de Saúde Pública Sergio Arouca, Fundação Oswaldo Cruz, Rio de Janeiro, Brasil.

Correspondência S. Lima

Escola Nacional de Saúde Pública Sergio Arouca, Fundação Oswaldo Cruz. Rua do Oriente 386, apto. 405, Rio de Janeiro, $R J$ 20240-130, Brasil. sheylla.lima@ensp.fiocruz.br
}

\begin{abstract}
Theoretical-conceptual models aim to integrate social, biological, behavioral, and other factors and to explain their interrelations in the determination of health outcomes. In the case of determination of neonatal death in Brazil, few studies have analyzed the mechanisms by which numerous variables and constructs interrelate. This article offers a theoretical discussion of the possible components of a hierarchical framework for studying neonatal mortality. The proposed model sought to include factors that have traditionally been identified as relevant to the occurrence of neonatal death and others that have been studied more recently (like race, social support, and violence during pregnancy). The components were distributed in 11 dimensions allocated to four hierarchical levels (one distal, two intermediary, and one proximal). The proposed framework should be adjusted to the Brazilian reality for neonatal death in large cities and raises various aspects for discussion that have not been explored previously in Brazil.
\end{abstract}

Theoretical Models; Neonatal Mortality (Public Health); Risk Factors
Sheylla de Lima 1

Márcia Lazaro de Carvalho 1

Ana Glória Godoi Vasconcelos 1

\section{Introdução}

A mortalidade neonatal resulta de uma estreita e complexa relação entre fatores de várias dimensões, exigindo a proposição de modelos hierarquizados complexos para o estudo de seus determinantes e inter-relações 1 .

Mosley \& Chen 2 foram os precursores dos modelos teórico-conceituais no campo da mortalidade infantil. Segundo esses autores, os fatores distais (antecedentes) influenciam os fatores intermediários e estes influenciam os fatores proximais (aqueles que agem de maneira mais direta sobre o desfecho). Assim, através de uma estrutura hierarquizada, é possível considerar e modelar fatores distintos de acordo com sua precedência no tempo e de sua relevância para a determinação do desfecho.

A abordagem estatística tradicional por meio de modelos de regressão múltipla, com apenas um nível hierárquico, não é a mais indicada em situações nas quais existem variáveis mediadoras ou intervenientes. Ao serem incluídas simultaneamente às distais, os efeitos daquelas variáveis podem ser mascarados ou subestimados. Quando excluídas, elos importantes de causalidade podem permanecer ignorados 3,4 .

No campo da modelagem multivariada, a incorporação de níveis hierárquicos diferenciados de determinação em relação ao desfecho tem sido respondida recorrendo-se aos chamados modelos hierarquizados 5,6. Nesses modelos, a 
mesma variável pode atuar como fator de confusão para fatores proximais e como mediadora para variáveis distais 4 .

Essa forma de posicionar as variáveis hierarquicamente, seja a partir da ordenação temporal ou lógica entre os eventos que conduzem ao desfecho, seja pela distinção conceitual em relação a uma variável de exposição como mediadoras ou de confusão, fornece um guia para análise e interpretação dos resultados à luz do conhecimento existente. Além disso, representa uma estratégia para lidar com um grande número de variáveis conceitualmente relacionadas, presentes em estudos epidemiológicos 3,7 .

O processo de análise estatística dos dados e interpretação dos resultados segue a coerência do modelo hierarquizado. Nessa estratégia de análise, a introdução das variáveis se dá em etapas, iniciando com as variáveis dos níveis mais distais e introduzindo-se simultaneamente apenas variáveis de um mesmo nível 3,5. O efeito de cada variável sobre o desfecho é interpretado como ajustado para as variáveis pertencentes aos níveis hierarquicamente anteriores (mais distais) e para os efeitos das variáveis que se encontram no mesmo nível. Assim, considerando-se um modelo com três níveis hierárquicos (distal, intermediário e proximal), o modelo de análise com variáveis dos três níveis apresenta estimativas dos efeitos das variáveis do nível distal no desfecho, não mediados por variáveis dos níveis seguintes; estimativas dos efeitos das variáveis do nível intermediário no desfecho, ajustadas por variáveis do nível distal e não mediados por variáveis do nível proximal, e estimativas dos efeitos das variáveis do nível proximal no desfecho ajustadas para variáveis dos níveis distal e intermediário.

O conhecimento a respeito do óbito neonatal necessita de aprofundamento em relação aos mecanismos por meio dos quais variáveis mediadoras (como por exemplo, apoio social e violência) conduzem os efeitos de indicadores sócio-econômicos sobre este desfecho. Adicionalmente, faz-se importante elucidar possíveis inter-relações entre variáveis ainda pouco investigadas nos estudos brasileiros (raça, apoio social e exposição à violência) e as já tradicionalmente conhecidas envolvidas na rede de causalidade deste evento, identificando o momento em que exercem maior impacto na sua ocorrência.

Políticas de saúde que visem a melhorar a cobertura e a qualidade da assistência durante o ciclo gravídico-puerperal têm impacto comprovado na magnitude da mortalidade neonatal $8,9,10$. No entanto, faz-se necessário reconhecer os fatores que influenciam o acesso a serviços de saúde, intervindo neles e elucidando os fatores estruturais que determinam o não-ingresso.
Estudos apontam como principais preditores para a ocorrência do óbito neonatal, fatores biológicos - peso ao nascer e idade gestacional, por exemplo - e outras condições de nascimento do recém-nascido, como o baixo escore de Apgar, comumente considerados no nível mais próximo ao desfecho 1,11,12,13,14. Variáveis que representam características maternas e de assistência à saúde são distribuídas nos níveis intermediários e apresentam efeitos cuja magnitude é reduzida quando incluídas as variáveis proximais $1,11,12$. Os fatores mais distais, em geral, referem-se às condições sócio-econômicas e demográficas da mãe e, na maioria das vezes, seus efeitos sobre o óbito neonatal deixam de ser estatisticamente significantes na presença de variáveis dos níveis intermediários e proximais $1,11,12,13$.

Este trabalho traz uma discussão teórica sobre os componentes que poderão compor um modelo hierarquizado para o estudo do óbito neonatal, fornecendo subsídios para o aprofundamento da discussão no que se refere aos mecanismos de mediação de efeitos das variáveis e sua inter-relação no desencadeamento de eventos que conduzem a este desfecho.

\section{Modelo hierarquizado: uma aplicação ao óbito neonatal}

O nível distal no modelo foi composto por características sócio-econômicas e demográficas maternas. Nos dois níveis intermediários, foram consideradas características maternas e aspectos referentes à assistência pré-natal e ao parto. Proximamente ao desfecho, incluíram-se fatores relacionados às condições de saúde e nascimento do recém-nascido e à assistência neonatal (Figura 1). A Figura 2 descreve esquematicamente as inter-relações entre as dimensões que compõem o nível referente às características maternas (nível intermediário I).

No modelo proposto, a idade materna foi considerada no nível intermediário, com o intuito de concebê-la não como característica puramente biológica, mas, sim, como característica que pode, de certo modo, sofrer influência de fatores situados no nível distal do modelo, como as condições sócio-econômicas. Alguns autores 8,15,16 observaram em seus estudos que a experiência de gravidez na adolescência é mais freqüente em grupos mais desfavorecidos social e economicamente. Adicionalmente, Sharma 17 aponta que as diferenças na mortalidade infantil por idade materna, mais do que o efeito biológico desta, podem refletir diferenças nas características sócio-econômicas das famílias. 


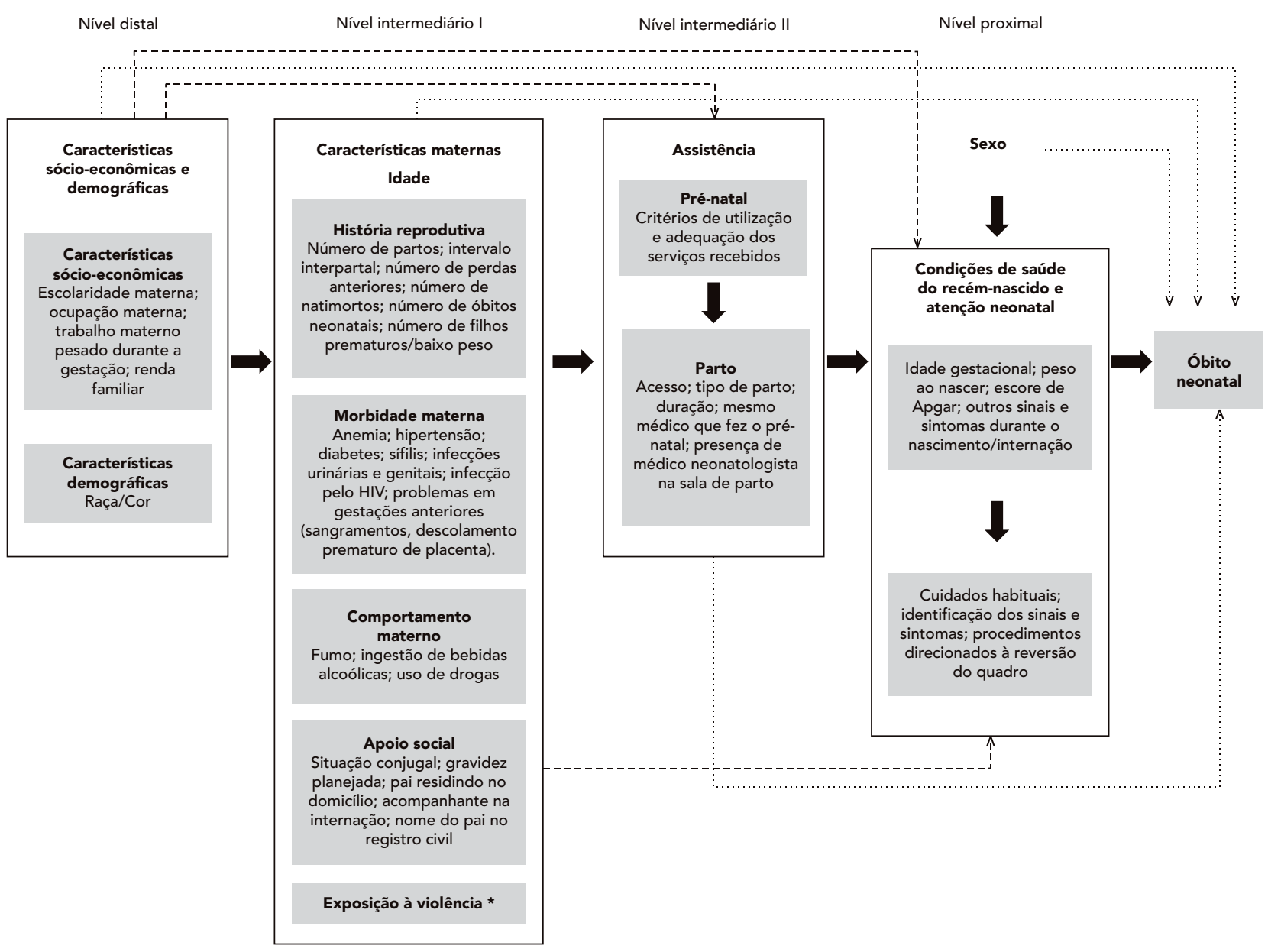

* Para se inferir sobre esta exposição devem ser utilizados instrumentos como o proposto por Reichenheim et al. 23.

O apoio social, a exposição à violência e os fatores relacionados ao comportamento materno, além de estarem intrinsecamente relacionados (setas de duplo sentido), como sugerem resultados de alguns estudos 8,15,18, de certa forma, antecedem ou determinam parcialmente a ocorrência de morbidades maternas e a história reprodutiva. Esses fatores podem ou não ter contribuído para os riscos relacionados às gestações anteriores, assim como aqueles que se apresentam na gestação atual. Por este motivo, a morbidade materna e a história reprodutiva estão dispostas mais próximas ao desfecho, pois são possíveis fatores de risco para a gravidez atual. Considerando-se ainda a possibilidade de a mulher não apresentar história reprodutiva anterior, por estar em sua primeira gravidez, esses fatores podem influenciar a ocorrência de óbito nas primeiras semanas de vida, em virtude, indiretamente, das morbidades maternas, e/ou desfechos como a prematuridade e o baixo peso ao nascer.

O nível intermediário II aponta a influência que características da assistência pré-natal exercem na assistência prestada no momento do parto. Um pré-natal de boa qualidade deverá ser capaz de garantir o acesso ao parto em unidade apropriada às necessidades identificadas nas consultas de pré-natal da parturiente, orientando a tomada de decisão em relação ao tipo 

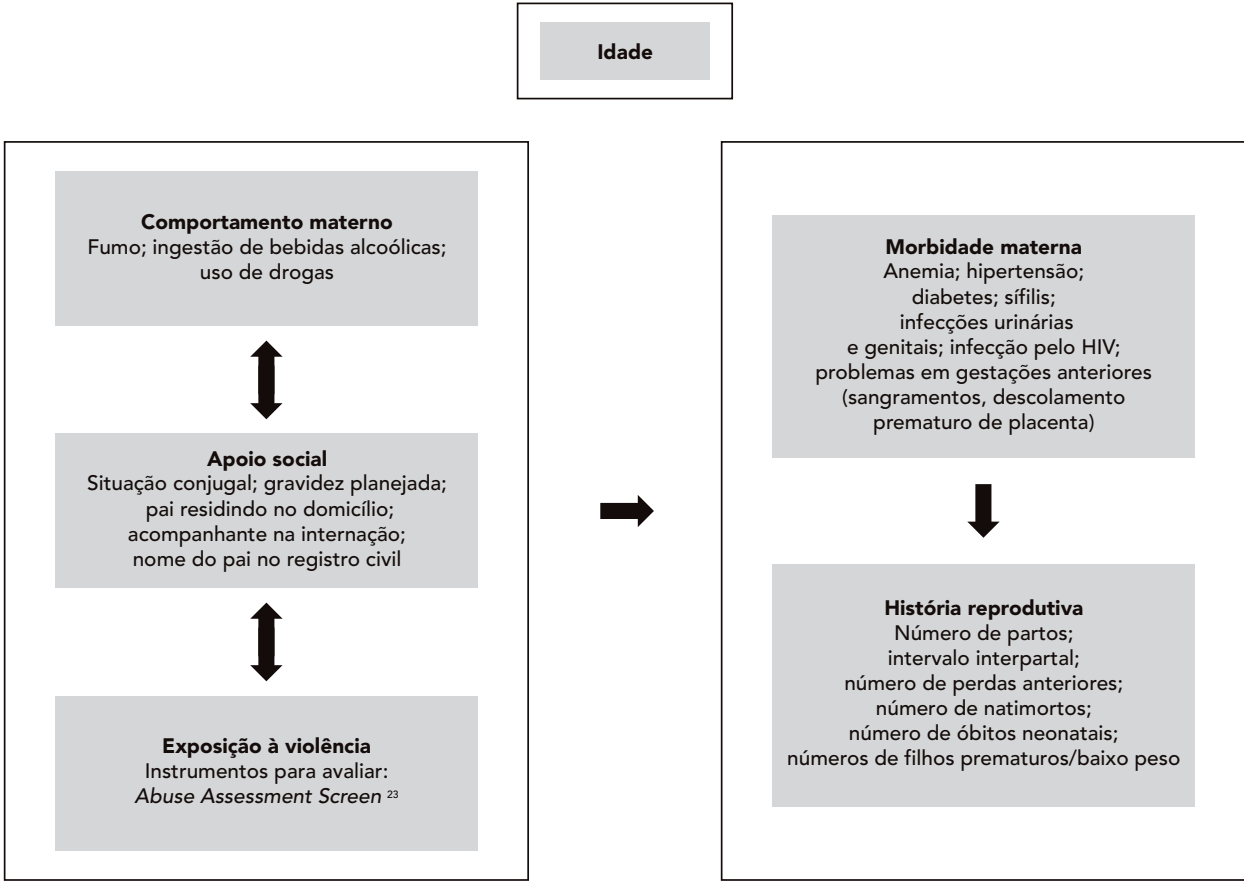

de parto mais indicado e demais condutas a serem adotadas em situações de risco para a dupla mãe-bebê.

Ainda no nível intermediário II, considerouse que certas características da atenção ao parto poderiam configurar-se em risco para o desfecho, como o fato de o parto não ser realizado pelo mesmo médico que acompanhou o pré-natal (este, pelo fato de conhecer a trajetória da gestante, poderia se antecipar às possíveis intercorrências no momento do parto) e a ausência de neonatologista na sala de parto para identificar, precocemente, a presença de sinais e sintomas anormais no recém-nascido.

As características de saúde do recém-nascido foram consideradas no nível proximal, por ser consenso a estreita relação que apresentam com o óbito infantil em seus distintos momentos. Entende-se que a assistência neonatal poderia reverter algumas características desfavoráveis do recém-nascido (como, por exemplo, o escore de Apgar no quinto minuto), desde que precoce e adequadamente diagnosticadas; portanto, considerou-se que estão correlacionadas (representação por seta larga unidirecional entre estas duas dimensões do nível proximal).

No modelo proposto, os efeitos indiretos entre os vários níveis estão representados. Há a possibilidade de que efeitos indiretos de fatores contemplados no nível distal se dêem em virtude da assistência ao pré-natal e ao parto, de algumas condições de saúde do recém-nascido e, especialmente, de características da atenção neonatal (setas unidirecionais tracejadas na Figura 1). Do mesmo modo, os efeitos indiretos das características maternas podem não ser mediados exclusivamente pelos fatores relacionados à assistência pré-natal e ao parto; a mediação pode se dar também pelas características do recémnascido, com exceção do sexo (seta unidirecional tracejada na Figura 1), situadas proximamente ao desfecho.

Os efeitos diretos no desfecho, exercidos por fatores situados no nível proximal, estão representados por seta unidirecional que conecta este nível ao desfecho. Entretanto, é possível que os 
fatores que compõem este nível (exceto o sexo) sejam mediadores de apenas parte dos efeitos indiretos dos fatores situados nos níveis distal e intermediários. Ou seja, fatores situados nos níveis mais distais deste modelo podem ainda apresentar efeitos diretos (setas unidirecionais pontilhadas na Figura 1) sobre o desfecho, não mediados pelas variáveis consideradas nos níveis intermediários e proximal.

Em relação ao nível proximal, é possível que o sexo do recém-nascido exerça efeitos diretos e indiretos sobre o óbito neonatal. Os efeitos indiretos estariam relacionados com peso ao nascer (seta larga unidirecional, que conecta esta característica do recém-nato às demais dimensões deste nível) ou com a ocorrência de morbidades específicas (não contempladas neste modelo). O possível efeito direto do sexo do recém-nascido sobre o óbito infantil neonatal (ainda controverso na literatura) encontra-se representado pela seta unidirecional pontilhada na Figura 1.

A variável sexo do recém-nascido no modelo hierarquizado proposto foi considerada no nível proximal, entendendo que, no início da vida, a contribuição de efeitos atribuídos à determinação biológica é predominante e mais expressiva 5 para a ocorrência de determinados eventos, como, por exemplo, o óbito neonatal.

Esta variável é independente de qualquer outro fator presente no modelo, não sendo intermediária em nenhum dos caminhos representados. A partir deste nível, o sexo da criança está iniciando mais um possível mecanismo causal.

\section{Comentários finais}

O modelo apresentado diferencia-se substancialmente dos já existentes por contemplar, além dos fatores de risco tradicionalmente apontados como envolvidos na cadeia causal que conduz ao desfecho, fatores ainda pouco explorados por estudos realizados no Brasil sobre o tema. Adicionalmente, aponta a necessidade de se estudarem e conhecerem as inter-relações e efeitos de mediação entre esses fatores, lacuna ainda pouco investigada por estudos que utilizam essa estratégia de modelagem.

Na lógica de construção do modelo hierarquizado proposto, utilizou-se o conceito de causas suficientes e componentes, que permite considerar a temporalidade e o sinergismo entre os fatores causais. Os fatores presentes nos distintos níveis representam causas componentes, podendo cada uma participar de um ou mais mecanismos causais. Nesse contexto, as inter-relações existentes entre os níveis de determinação seriam os chamados mecanismos ou cadeias causais, podendo cada uma destas cadeias ser uma causa suficiente para a ocorrência do desfecho ${ }^{5}$. No modelo, poder-se-ia mencionar, a título de exemplo: mulheres com características sócio-econômicas (nível distal) desfavoráveis apresentam menor autonomia de escolha do local do parto e maior chance de ingressar em serviços de saúde que têm condições precárias de assistência (nível intermediário II). Isso, por sua vez, pode interferir nas condições de nascimento do bebê, bem como na assistência neonatal prestada (nível proximal), o que, finalmente, poderia aumentar a chance de óbito neonatal.

A hierarquização dos níveis também representa a ordem cronológica dos eventos e facilita a identificação de elos da cadeia causal onde as intervenções podem ser aplicadas. Intervenções de longo e médio prazo referem-se, em geral, a questões estruturais como educação e renda, que têm efeitos indiretos sobre o desfecho.

Nos níveis intermediários, foram incluídos fatores de mediação como aqueles relativos à assistência, nos quais determinantes distais exercem seus efeitos. A ampliação do acesso e a melhora da qualidade dos serviços de saúde podem, até certo ponto, contribuir para a redução dos níveis de mortalidade neonatal. Mas faz-se importante ainda representar a cadeia de causalidade englobando os diversos fatores que contribuem para a ocorrência deste desfecho, no intuito de se explicar que condições sócio-econômicas permanecem sendo as principais causas do processo que culmina no óbito neonatal. Assim, evita-se, por exemplo, culpar a mãe por comportamentos não saudáveis durante a gravidez.

Na avaliação da atenção pré-natal, o modelo aponta para que se considerem aspectos quantitativos, devidamente ajustados para a idade gestacional, acrescidos de critérios que avaliem a qualidade e adequação dos cuidados recebidos, pelos motivos já apontados na literatura 9,17: (1) evitar subestimar o efeito protetor do pré-natal, uma vez que desfechos adversos são comumente associados a gestações curtas, e, conseqüentemente, ao menor número de consultas realizadas, e (2) valorizar não só a captação precoce, mas também a continuidade dos contatos e intervenções efetivas.

No nível proximal, a hipótese norteadora é a de que as características de saúde e nascimento exercem efeitos diretos sobre a chance de sobrevivência infantil, sendo, além disso, expressões de efeitos indiretos exercidos pelas condições de vida, saúde e de assistência anteriores à sua ocorrência. Adicionalmente, a assistência neonatal desempenharia importante papel no que se refere aos cuidados imediatos ao recém-nascido, 
sobretudo aos que apresentaram condições de saúde desfavoráveis ao nascer.

O modelo proposto representa, hierarquicamente, os vários fatores envolvidos na cadeia de causalidade que conduzem ao óbito infantil neonatal e as possíveis inter-relações que se estabelecem entre eles. Todavia, como todo modelo, tenta "simplificar" uma realidade muito complexa, não se propondo a ser um modelo ideal. Espera-se que possa ser testado - após adequação às características locais - e que venha contribuir para o entendimento dos fatores e mecanismos que acarretam o óbito no período neonatal, de modo que tal compreensão possa ser empregada na adoção de medidas de intervenção, a fim de reduzir as desigualdades em saúde e prevenir características desfavoráveis do recém-nascido.

Embora o modelo proposto não tenha sido testado, pode-se sugerir que o método de análise a ser empregado no ajuste seja a análise multivariada por modelos de regressão, adotando a técnica de níveis hierárquicos. Esta abordagem representa um avanço em relação à modelagem de regressão convencional, com apenas um nível hierárquico, por permitir a inclusão de variáveis em diferentes níveis e, conseqüentemente, considerações relativas à ordenação temporal ou lógica entre os fatores e hipóteses de interme- diação de efeitos. Contudo, esta técnica apresenta limitações metodológicas, destacando-se os seguintes aspectos: a não-estimação dos efeitos indiretos, representação limitada das inter-relações entre os diversos níveis hierárquicos e a não-obtenção de índices globais de ajuste 4,7 .

Estimativas de efeitos indiretos em análises envolvendo constructos podem ser obtidas por meio dos modelos de equações estruturais, uma técnica de análise multivariada amplamente conhecida nas áreas de ciências sociais e psicologia, mas ainda pouco utilizada na epidemiologia 19 . Esses modelos acomodam as limitações citadas anteriormente, permitindo lidar simultaneamente, e de forma mais eficiente, com um grande número de variáveis e suas inter-relações 7,20,21. Esta estratégia de modelagem pode ser considerada, no contexto de análises hierarquizadas, como o modelo proposto.

No caso de estudos que considerem outras estruturas de interdependência na produção do óbito neonatal (como, por exemplo, município, comunidade, hospitais), a análise multinível pode ser uma técnica estatística a ser utilizada no ajuste. Esta modelagem, além de identificar a variabilidade atribuída a cada nível na determinação do evento estudado, também lida com o efeito de cluster, ou seja, a não-independência das observações 22 .

\section{Resumo}

Modelos teórico-conceituais visam a integrar fatores sociais, biológicos, de comportamento, entre outros, $e$ ainda elucidar as relações que se estabelecem entre eles na determinação de desfechos de saúde. No caso da determinação do óbito neonatal em nosso meio, poucos estudos analisam os mecanismos por meio dos quais as inúmeras variáveis e constructos se inter-relacionam. Este trabalho traz uma discussão teórica sobre os componentes que poderão compor um modelo hierarquizado para o estudo do óbito neonatal. Na proposição do modelo, buscou-se incluir fatores tradicionalmente apontados como importantes para a ocorrência desse evento e fatores que vêm sendo recentemente estudados (como raça, apoio social e violência durante a gravidez). Os componentes foram distribuídos em 11 dimensões alocadas em quatro níveis hierárquicos (um distal, dois intermediários e um proximal). O modelo proposto deve ser adequado à realidade brasileira referente ao óbito neonatal em grandes centros urbanos e traz à discussão aspectos ainda não muito explorados por estudos realizados no Brasil.

Modelos Teóricos; Mortalidade Neonatal; Fatores de Risco

\section{Colaboradores}

S. Lima idealizou e redigiu o artigo. M. L. Carvalho e A. G. G. Vasconcelos idealizaram o artigo e coordenaram a sua elaboração. 


\section{Referências}

1. Almeida SDM, Barros MBA. Atenção à saúde e mortalidade neonatal: estudo caso-controle realizado em Campinas, São Paulo. Rev Bras Epidemiol 2004; 7:22-35.

2. Mosley WH, Chen LC. An analytical framework for the study of child survival in developing countries. Popul Dev Rev 1984; 10 Suppl:25-45.

3. Victora CG, Huttly SR, Fuchs SC, Olinto MTA. The role of conceptual frameworks in epidemiological analysis: a hierarchical approach. Int J Epidemiol 1997; 26:224-7.

4. Vasconcelos AGG, Almeida RMV, Nobre FF. Path analysis and multi-criteria decision making: an approach for multivariate model selection and analysis in health. Ann Epidemiol 2001; 11:377-84.

5. Olinto MTA. Reflexões sobre o uso do conceito de gênero e/ou sexo na epidemiologia: um exemplo nos modelos hierarquizados de análise. Rev Bras Epidemiol 1998; 1:161-9.

6. Albernaz EP, Menezes AMB, César JA, Victora CG, Barros FC, Halpern R. Fatores de risco associados à hospitalização por bronquiolite aguda no período pós-neonatal. Rev Saúde Pública 2003; 37:485-93.

7. Vasconcelos AG, Almeida RMV, Nobre FF. The path analysis approach for the multivariate analysis of infant mortality data. Ann Epidemiol 1998; 8:262-71.

8. Gama SNG, Szwarcwald CL, Leal MC. Experiência de gravidez na adolescência, fatores associados e resultados perinatais entre puérperas de baixa renda. Cad Saúde Pública 2002; 18:153-61.

9. Coimbra CL, Silva AAM, Mochel EG, Alves MTSSB, Ribeiro VS, Aragão VMF, et al. Fatores associados à inadequação do uso da assistência pré-natal. Rev Saúde Pública 2003; 37:456-62.

10. Gomes MASM, Lopes JMA, Moreira MEL, Gianini NOM. Assistência e mortalidade neonatal no setor público do Município do Rio de Janeiro, Brasil: uma análise do período 1994/2000. Cad Saúde Pública 2005; 21:1269-77.

11. Almeida MF, Novaes HMD, Alencar GP, Rodrigues, LC. Mortalidade neonatal no Município de São Paulo: influência do peso ao nascer e de fatores sócio-demográficos e assistenciais. Rev Bras Epidemiol 2002; 5:93-107.

12. Morais Neto OL, Barros MBA. Fatores de risco para mortalidade neonatal e pós-neonatal na Região Centro-Oeste do Brasil: linkage entre bancos de dados de nascidos vivos e óbitos infantis. Cad Saúde Pública 2000; 16:477-85.
13. Martins EF, Velásquez-Meléndez G. Determinantes da mortalidade neonatal a partir de uma coorte de nascidos vivos, Montes Claros, Minas Gerais, 1997-1999. Rev Bras Saúde Matern Infant 2004; 4:405-12.

14. Menezes AMB, Barros FC, Victora CG, Tomasi E, Halpern R, Oliveira ALB. Fatores de risco para mortalidade perinatal em Pelotas, RS, 1993. Rev Saúde Pública 1998; 32:209-16.

15. Sabroza AR, Leal MC, Gama SGN, Costa JV. Perfil sócio-demográfico e psicossocial de puérperas adolescentes do Município do Rio de Janeiro, Brasil - 1999-2001. Cad Saúde Pública 2004; 20 Suppl 1: S112-20.

16. Gama SNG, Szwarcwald CL, Leal MC, Theme Filha MM. Gravidez na adolescência como fator de risco para baixo peso ao nascer no Município do Rio de Janeiro, 1996 a 1998. Rev Saúde Pública 2001; 35:74-80.

17. Sharma RK. Causal pathways to infant mortality: linking social variables to infant mortality through intermediate variables. J Health Soc Policy 1998; 9:15-28.

18. Moraes CL, Reichenheim ME. Domestic violence during pregnancy in Rio de Janeiro, Brazil. Int J Gynaecol Obstet 2002; 79:269-77.

19. Kline RB. Principles and practice of structural equation modeling. New York: Guilford; 1998.

20. Kiely JL. Some conceptual problems in multivariable analyses of perinatal mortality. Paediatr Perinat Epidemiol 1991; 5:243-57.

21. Ribeiro VS, Silva AAM, Barbieri MA, Bettiol H, Aragão VMF, Coimbra LC, et al. Infant mortality: comparison between two birth cohorts from Southeast and Northeast, Brazil. Rev Saúde Pública 2004; 38:773-9.

22. Carvalho MS, Andreozzi VL, Codeço CT, Barbosa MTS, Shikamura SE. Análise de sobrevida: teoria e aplicações em saúde. Rio de Janeiro: Editora Fiocruz; 2005.

23. Reichenheim ME, Moraes CL, Hasselman MH. Equivalência semântica da versão em português do instrumento Abuse Assessment Screen para rastrear a violência contra a mulher grávida. Rev Saúde Pública 2000; 34:610-6.

Recebido em 14/Fev/2007

Versão final reapresentada em 10/Out/2007

Aprovado em 10/Jan/2008 